\title{
SIMPLE NEURAL MODEL FOR APPROXIMATE LOCALIZATION OF MOBILE STOCHASTIC EM SOURCES WITH VARIABLE SIGNAL LEVEL AND LINEAR TRAJECTORY IN THE AZIMUTHAL PLANE
}

\author{
Ivan Milovanovic, ${ }^{1, *}$ \\ Zoran Stankovic ${ }^{2}$, \\ Nebojsa Doncov², \\ Bratislav Milovanovic ${ }^{1}$
}

\author{
'Singidunum University, \\ Belgrade, Serbia \\ 2University of Niš, \\ Faculty of Electronic Engineering, \\ Niš, Serbia
}

Correspondence:

Ivan Milovanović

e-mail:

imilovanovic@singidunum.ac.rs

\begin{abstract}
:
In this paper, a simple neural model is presented for approximate determination of the angular position of stochastic radiation sources that move along the linear trajectory at azimuthal plane and whose incoming signal level fluctuates with the change of their position. The neural model is based on a multilayer perceptron network (MLP). In order to perform the localization of stochastic sources, the signals of these sources are sampled with a linear uniform antenna array, and then based on the output of the antenna sensor, spatial correlation matrix is formed, and at the end, the values of this matrix are input to the MLP network determining the angular positions of the source.
\end{abstract}

Keywords:

Stochastic EM sources, DoA estimation, Neural networks, Neural model.

\section{INTRODUCTION}

Either deterministic or stochastic radiation presence in the sources of electromagnetic interference (EMI), can degrade the performance of today's wireless communication systems, gravely [1-3]. The negative impact can be muffled by a spatial signal filtering using adaptive antenna arrays [1]. Adaptive beamforming of such antenna arrays is usually based on the Direction of Arrival (DoA) estimation techniques commonly based on the super-resolution algorithms such as MUSIC and its modifications $[1,4]$. Implementing these algorithms may be faced with hardware constraints of different types so they may not be applicable for real-time applications. Therefore, the usage of artificial neural networks (ANNs) in the DoA estimation process was proposed in [5-7] as a good real-time alternative.

Multilayer perceptron (MLP) neural networks were used by the authors of this paper for the realization of a neural model for 2D DoA estimation of deterministic [8] and 1D DoA estimation of stochastic sources of electromagnetic (EM) radiation $[9,10]$. For stochastic EMI, either uncorrelated [9] or partially correlated sources [10] were taken into consideration. It was presented that in both stochastic cases, it is sufficient enough to take just the values of the first row of spatial correlation matrix obtained by an antenna array sampling, for the training of a 
neural model that provides 1D DoA estimation with an accuracy close to super-resolution algorithms but with much faster run-time.

In all previous cases [9-10], it was assumed the stochastic EM sources are with constant radiation power during their movement. In [11] we consider for the first time, the more general scenario in which movable stochastic EM sources are with fluctuated incoming signal level (ISL). Cascade two-stage neural model is proposed to efficiently perform 1D localization of such mobile stochastic EM sources in ideal conditions, when noise is not present.

This paper presents an alternative neural network solution for the same problem of spatial localization considered in [11]. Unlike [11] where a complex two-stage neural model is used, a simpler MLP model is used to solve the problem, which is less accurate than the model in [11], but it therefore has a simpler architecture and is easier to train and implement. The model proposed in this paper is suitable for use in conditions where high precision is not required in determining the spatial position of stochastic sources, and when noise is collected by antenna series is very small so it can be ignored.

\section{STOCHASTIC SOURCE RADIATION MODEL}

Our Initial assumption in research presented in this, as in our previous papers [9-10], is that a stochastic EM source in the far-field can be represented by a short dipole, fed by stochastic current. Sources are moving in the azimuth plane along the straight $1 \mathrm{D}$ path and the orientation of each dipole representing that one source can change during the movement, allowing us to simulate the fluctuation of stochastic source radiation power in time. Equal level of correlation between the stochastic EM sources is expressed through the correlation between fed currents of dipoles as [2,3]:

$\mathbf{c}^{I}(\omega)=\lim _{T \rightarrow \infty} \frac{1}{2 T}\left[\mathbf{I}(\omega) \mathbf{I}(\omega)^{H}\right]$

Where is $\mathbf{I}=\left[I_{1}, I_{2}, \ldots, I_{S}\right]^{\mathrm{T}}$ a vector of fed current of dipoles and $S$ is the number of stochastic EM sources. Using the Green function in free space we get:

$$
\begin{aligned}
& \mathbf{M}^{(\mathrm{A})}=\mathbf{M}^{(\mathrm{A})}\left(\theta_{1}, \ldots, \theta_{s}, \ldots, \theta_{S}, \phi_{1}, \ldots, \phi_{s}, \ldots, \phi_{S}, r_{1}, \ldots, r_{s}, \ldots, r_{S}\right)= \\
& \frac{j z_{0}}{2 \pi}\left[\frac{F_{1}\left(\theta_{1}, \phi_{1}\right) e^{j k k_{1}}}{r_{1}} \ldots \frac{F_{s}\left(\theta_{s}, \phi_{s}\right) e^{j k r_{s}}}{r_{s}} \ldots \frac{F_{S}\left(\theta_{S}, \phi_{S}\right) e^{j k_{s}}}{r_{S}}\right]
\end{aligned}
$$

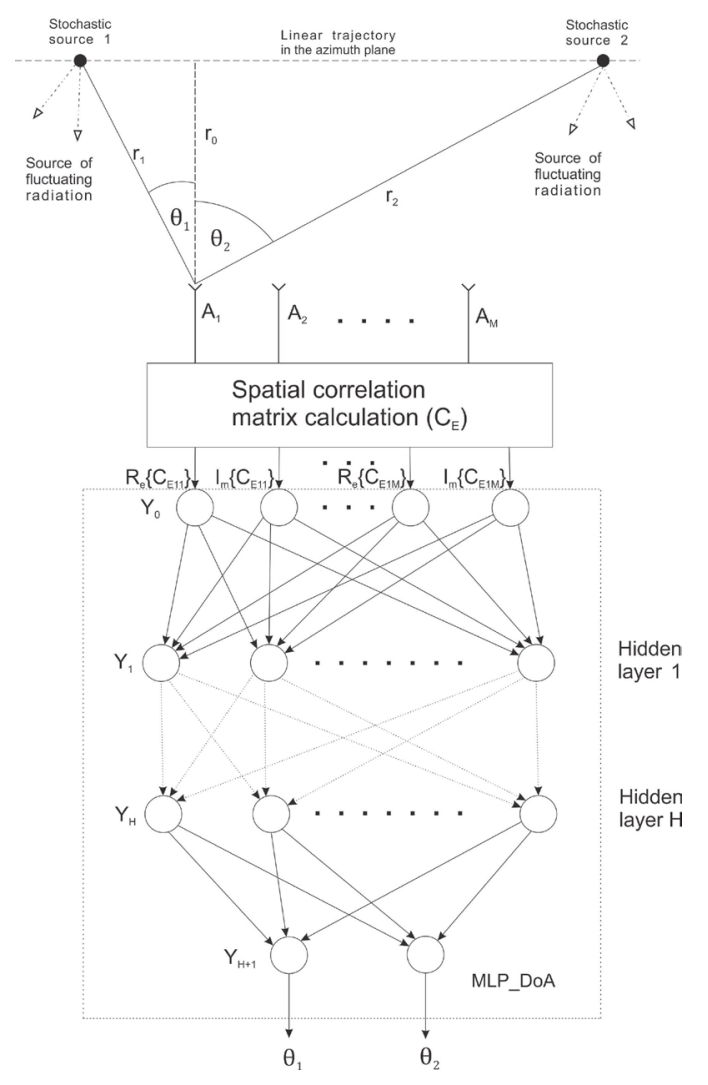

Fig 1. Angular position of mobile stochastic EM sources with fluctuating radiation with respect to the sensors of antenna array performing the sampling of the received signals and architecture of MLP_DoA neural model 
The electric field in a specific sampling point $A$ in the far-field, can be calculated as:

$E^{(\mathrm{A})}\left(\theta_{1}, \ldots, \theta_{s}, \ldots \theta_{s}, \varphi_{1}, \ldots, \varphi_{s}, \ldots \varphi_{S}, r_{1}, \ldots, r_{s}, \ldots r_{S}\right)=\mathbf{M}^{(\mathrm{A})} \mathbf{I}$

In the Eq. $(2), F_{s}(\theta, \varphi)$ is the radiation pattern of the $s$-th stochastic EM source, $r_{s}$ is distance between the $s$-th stochastic EM source and the sampling point. $z_{0}$ is the impedance of free space, $k$ is the phase constant $(k=2 \pi / \lambda)$, while $\theta$ and $\varphi$ are angles used to describe the angular position of stochastic EM source relative to the particular sampling point in the far-field.

Spatial correlation matrix in the far field is acquired by sampling the signals using a uniform linear antenna array with $M$ sensors (Fig. 1, the shown scenario of movement and DoA estimation of two stochastic sources). Axis of the antenna array is parallel to the direction of movement of stochastic EM sources, while the sensor positions are described by points $A_{1}, A_{2}, \ldots, A_{M^{*}}$. Sensors are at equal mutual distance $y_{\mathrm{d}}$.

Combining Eqs. (1), (2) and (3), the correlation matrix in the far field is expressed as:

$$
\widetilde{\mathbf{C}}_{E}[i, j]=\mathbf{M}^{\left(\mathrm{A}_{i}\right)} \mathbf{c}^{I}\left(\mathbf{M}^{\left(\mathrm{A}_{j}\right)}\right)^{H} \quad i=1, \ldots, M j=1, \ldots, M
$$

As it was initially assumed, EM sources are moving only in azimuth plane, angle $\varphi$ is equals zero for any position of the stochastic EM source and the matrix $\mathbf{c}^{I}$, is a unit matrix due to uncorrelated sources. Therefore, the azimuth angular position and the distance of $s$-th stochastic EM source relative to $m$-th antenna array sensor is [11]:

$$
\theta_{s}^{(m)}=\arctan \left[\tan \theta_{s}^{(1)}-\frac{(m-1) \cdot y_{d}}{r_{0}}\right] r_{s}^{(m)}=\frac{r_{0}}{\cos \theta_{s}^{(m)}}
$$

Angle $\theta_{s}$ represents the azimuth angular position of $s$-th source relative to the antenna array and it can be determined as an angle position in relation to the first sensor of the antenna array when $\theta_{s}=\theta_{s}^{(1)}$. Using Eqs. (2), (4) and (5) for the given angular position of the radiation source we may determine the mapping function $\mathbf{M}$, and after that also the elements of the correlation matrix using the Eq. (4). Fig.1 illustrates movement of the two stochastic EM sources $(S=2)$ with variable radiated power. For development of the neural model, we used the correlation matrix, normalized with the first matrix element:

$$
\mathbf{C}_{E}=\frac{1}{\tilde{C}_{E 11}} \cdot \tilde{\mathbf{C}}_{E}
$$

In this scenario, the changes in radiation power of $s$-th stochastic source are modeled through a variable relative ratio of radiation pattern functions:

$$
p_{s}\left(\theta_{s}, \phi_{s}\right)=10 \log \left[\frac{F_{s}\left(\theta_{s}, \phi_{s}\right)}{F_{1}\left(\theta_{1}, \phi_{1}\right)}\right]
$$

\section{NEURAL NETWORK MODEL}

A neural model based on a single MLP network along with its architecture and connection to the antenna array is shown in Figure 1. As shown in $[8,9,11]$, it is sufficient to consider only the elements of the first row of the correlation matrix $\mathrm{CE}$ for the training of the MLP model, performing 1D DoA estimation of uncorrelated radiation sources whose ISL is fluctuated during the movement. Accordingly, the fundamental task of the MLP_DoA model is to map the first row of the correlation matrix $\mathbf{x}\left(\mathbf{C}_{E}\right)=\left[C_{j}\right]_{1 \times M}, i=1, j \in\{1, \ldots, M\}$, sampled under the condition of variable radiation power of stochastic sources during their movement, to the azimuth position of stochastic sources:

$\left[\theta_{1} \theta_{2}\right]^{T}=f_{M L P \_D o A}\left(\mathbf{x}\left(\mathbf{C}_{E}\right)\right)$

MLP networks used in the MLP_DoA neural model is described by the following function:

$\mathbf{y}_{l}=F\left(\mathbf{w}_{l} \mathbf{y}_{l-1}+\mathbf{b}_{l}\right) \quad l=1,2, \ldots, H$

where $\mathbf{y}_{l-1}$ vector represents the output of (l-1)-th hidden layer, $\mathbf{w}_{l}$ is a connection weight matrix among $(l-1)$-th and $l$-th hidden layer neurons, $\mathbf{b}_{l}$ is a vector containing biases of $l$-th hidden layer neurons and $H$ is number of hidden layers. $F$ is the activation function of neurons in hidden layers and in this scenario it is a hyperbolic tangent sigmoid transfer function $F(u)=\left(e^{u}-e^{-u}\right) /\left(e^{u}-e^{-u}\right)$. Real and imaginary parts of correlation matrix elements are individually brought at the input point of the network. Therefore, the input layer of MLP network is in the form of: $\mathbf{y}_{0}=\left[\operatorname{Re}\left\{\mathbf{x}\left(\mathbf{C}_{E}\right)\right\} \mathrm{m}\left\{\mathbf{x}\left(\mathbf{C}_{E}\right)\right\}\right]_{1 \times 2 M}$, while output of the MLP_DoA network model is: $\left[\theta_{1} \theta_{2}\right]^{T}=\mathbf{w}_{H+1} \mathbf{y}_{H}$.

In these notations $\mathbf{w}_{H+1}$ connects in weight matrix between neurons of the last hidden layer and neurons in the output layer. During the training of the neural network, weight matrices $\mathbf{w}_{1}, \mathbf{w}_{2}, \ldots, \mathbf{w}_{H}, \mathbf{w}_{H+1}$ and biases values are optimized to get the desired mapping accuracy. The general notation used to describe the 
MLP neural model in the first or second model stage is: MLPH- $N_{1}-\ldots-N_{i}-\ldots-N_{H}$ where $H$ is the total number of hidden MLP network layers, and $N_{i}$ is the total number of neurons in the $i$-th hidden layer.

\section{MODELING RESULTS}

A MLP neural model, whose architecture is shown in the Fig. 1, is used to determine the angular position in the azimuth plane of two uncorrelated stochastic EM sources with fluctuated ISL. Sources are moving along a straight line trajectory that is outlying from the sampling place at $r_{0}=1000 \mathrm{~m}$. Parameter $p$, gives information about the change of difference between signal level of two sources, varies in the range $0 \mathrm{~dB} \leq p \leq 10 \mathrm{~dB}$. The signal is sampled by the antenna array consisting of, $M=4$ sensor elements at the frequency of $f=28 \mathrm{GHz}$. The distance between the neighboring antenna sensors is $y_{\mathrm{d}}=0.51$. Implementation, training and testing of neural models were conducted in MATLAB software environment. Method used for training is Quasi-Newton, with the prescribed accuracy of $10^{-4}$.

Based on relations presented in Eqs (2), (4) and (7), an inverse mapping is established here:

$\mathbf{C}_{E}^{t}=f_{D o A}^{-1}\left(\theta_{1}^{t}, \theta_{1}^{t}, p\right)$

Table 1. Testing results for six MLP_DoA neural networks with the best test statistics

\begin{tabular}{cccc}
\hline MLP network & $\begin{array}{c}\text { WCE } \\
{[\%]}\end{array}$ & $\begin{array}{c}\text { ATE } \\
{[\%]}\end{array}$ & $\boldsymbol{r}^{P P M}$ \\
\hline MLP_DoA_2-23-23 & 10.23 & 0.95 & 0.9984 \\
\hline MLP_DoA_4-22-20 & 9.97 & 0.97 & 0.9983 \\
\hline MLP_DoA_4-22-22 & 10.87 & 0.98 & 0.9982 \\
\hline MLP_DoA_4-18-18 & 10.68 & 1.02 & 0.9982 \\
\hline MLP_DoA_4-18-16 & 11.01 & 1.06 & 0.9980 \\
\hline MLP_DoA_4-18-14 & 10.45 & 1.12 & 0.9977 \\
\hline
\end{tabular}

By using this mapping, the training and testing sets for MLP networks are generated and they are in the form $\left\{\left(\mathbf{x}^{\mathrm{t}}\left(\mathbf{C}_{\mathrm{E}}^{\mathrm{t}}\left(\theta_{1}^{\mathrm{t}}, \theta^{\mathrm{t}}, p^{\mathrm{t}}\right)\right), \theta_{1}^{\mathrm{t}}, \theta_{2}^{\mathrm{t}}\right)\right\}$. Thereby the following distribution of samples is used [11]

$$
\left\{\begin{array}{l}
\left(\mathbf{x}^{t}\left(\mathbf{C}_{E}^{t}\left(\theta_{1}^{t}, \theta_{2}^{t}, p^{t}\right)\right), \theta_{1}^{t}, \theta_{2}^{t}\right) \mid \\
\theta_{1}^{t} \in\left[-30: \theta_{\text {step }}^{t}: 30\right], \theta_{2}^{t} \in\left[-30: \theta_{\text {step }}^{t}: 30\right], \theta_{1}^{t}>\theta_{2}^{t}, \\
c^{t} \in\left[0: p_{\text {step }}^{t}: 10\right]
\end{array}\right\}
$$

where values used to generate training and testing sets are $\theta_{\text {step }}^{\mathrm{t}}=1, p_{\text {step }}^{\mathrm{t}}=1$ and $\theta_{\text {step }}^{\mathrm{t}}=1.3, p_{\text {step }}^{\mathrm{t}}=1.3 \mathrm{respec}$ tively. 20130 training samples and 8648 testing samples were generated by using Eqs. (10) and (11). For the development of the model, networks with two hidden layers were used. Accordingly, using the training set, training of a number of MLP2-N1-N2 networks with variable number of N1 and N2 neurons in hidden layers was carried out. After testing all trained grids, the selection of the appropriate grids had the best test characteristics. Testing results for six MLP_DoA neural networks with the best test statistics are shown in Table 1. Testing metrics is WCE - worst case error, ACE - average test error and correlation coefficient rPPM [6]

For modeling, the MLP_DoA_2-23-23 network was selected to show the best test characteristics. Scattering diagram of the MLP_DoA model realized by using MLP_DoA_2-23-23 networks is shown in Fig. 2.

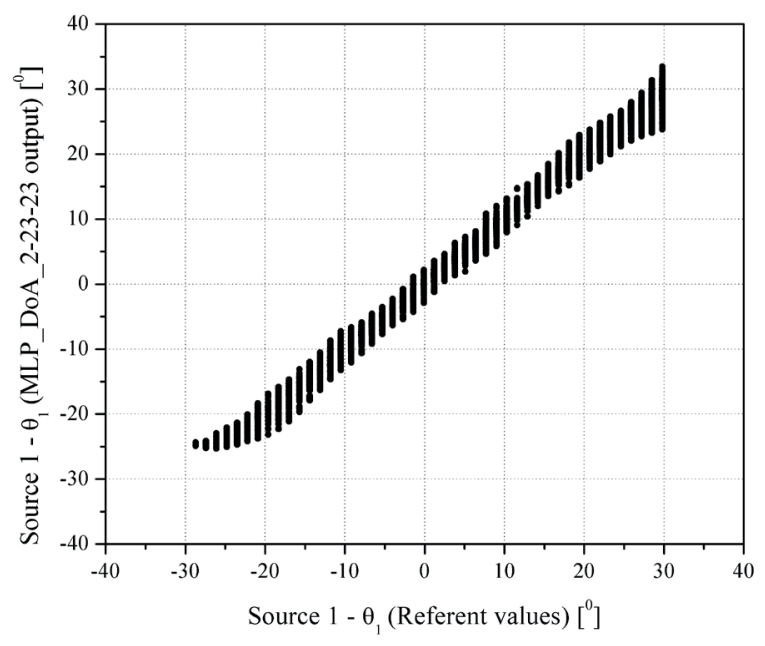

(a)

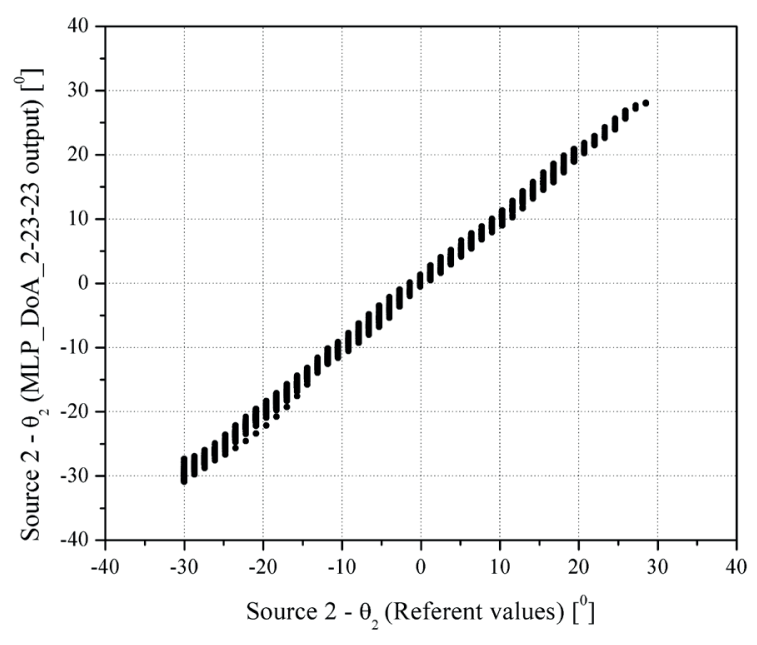

b)

Fig 2. Scattering diagram of MLP_DoA_2-23-23 model on test set: (a) $\theta_{1}$ output and (b) $\theta_{2}$ output 


\section{CONCLUSION}

The proposed neural model can be a good alternative to other neural models that deal with 1D DoA estimation and spatial localization of stochastic EM sources in the azimuthal plane that have a source of fluctuating radiation in the case when the priority is not the high accuracy of the model but its training speed and simple implementation. The model avoids complex mathematical calculations that are present in classical superresolution algorithms and hence has a very high simulation rate. The model is only applicable when the noise collected by the antenna array is very minor and can be ignored.

\section{ACKNOWLEDGMENT}

This work has been supported by the Ministry for Education, Science and Technological Development of Serbia, projects number TR32052 and TR32054.

\section{REFERENCES}

[1] B. Allen, M. Ghavami, Adaptive Array Systems: fundamentals and applications, Wiley, 2005

[2] J.A. Russer, T. Asenov and P. Russer, "Sampling of stochastic electromagnetic fields", IEEE MTT-S International Microwave Symposium Digest, Montreal, Canada, pp. 1-3, 2012.

[3] J.A. Russer, P. Russer, "Modeling of Noisy EM Field Propagation Using Correlation Information", IEEE Transactions on Microwave Theory and Techniques, Volume 63, Issue 1, pp.76-89, 2015.
[4] R. Schmidt, "Multiple emitter location and signal parameter estimation", IEEE Transactions on Antennas and Propagation, vol. 34, no. 3, pp. 276-28, 1986.

[5] S. Haykin, Neural Networks, New York, IEEE, 1994.

[6] Q. J. Zhang, K. C. Gupta, Neural networks for RF and microwave design, Artech House, Boston, MA, 2000.

[7] A. H. El Zooghby, C. G. Christodoulou, M. Georgiopoulos, "A neural network based smart antenna for multiple source tracking", IEEE Trans. on Antennas and Propagation, Vol. 48, no. 5, pp. 768 776, 2000.

[8] M. Agatonović, Z. Stanković, N. Dončov, L. Sit, B. Milovanović, T. Zwick, "Application of artificial neural networks for efficient high-resolution 2D DOA estimation", Radioengineering, Vol. 21, No. 4, pp. 1178-1186, 2012.

[9] Z. Stankovic, N. Doncov, I. Milovanović, B. Milovanović, "Neural network model for efficient localization of a number of mutually arbitrary positioned stochastic EM sources in far-field", Proc. of the 12th Symposium on Neural Network Applications in Electrical Eng. - NEUREL 2014, Beograd, Serbia, pp. 41-44, 2014.

[10] Z. Stanković, N. Doncov, I. Milovanović, M. Sarevska, B. Milovanović, "Neural Model for Far-Field 1D Localization of Mobile Stochastic EM Sources with Partially Correlated Radiation", Proceedings of the International Scientific Conference on Information Technology and Data Related Research - SINTEZA 2017, Belgrade, Serbia, pp. 169-175, 2017.

[11] Z. Stanković, N. Dončov, I. Milovanović, B. Stošić, B. Milovanović, J. Russer, "1D Localization of Mobile Stochastic EM Sources with Variable Radiated Power using Two-Stage Neural Model", 2nd URSI Atlantic Radio Science Conference (URSI AT-RASC), 2018, doi: 10.23919/URSI-AT-RASC.2018.8471456. 